\title{
A Review of Time Critical Decision Making Models and Human Cognitive Processes
}

\author{
Ron Azuma, Mike Daily, Chris Furmanski \\ HRL Laboratories, LLC \\ 3011 Malibu Canyon Rd. \\ Malibu, CA 90265 \\ 310-317-5451,310-317-5673 \\ azuma@hrl.com, daily@hrl.com,chris@furmanski.net
}

\begin{abstract}
From a general cognitive perspective, decision making is the process of selecting a choice or course of action from a set of alternatives. A large number of time critical decision making models have been developed over the course of several decades. This paper reviews both the underlying cognitive processes and several decision making models. In the first section, we briefly describe the primary underlying cognitive processes and issues that are common to most, if not all, decision making models, with a focus on attention, working memory, and reasoning. The second section reviews several of the most prominent high-level models of decision making, especially those developed for military contexts. ${ }^{12}$

\section{TABLE OF CONTENTS}

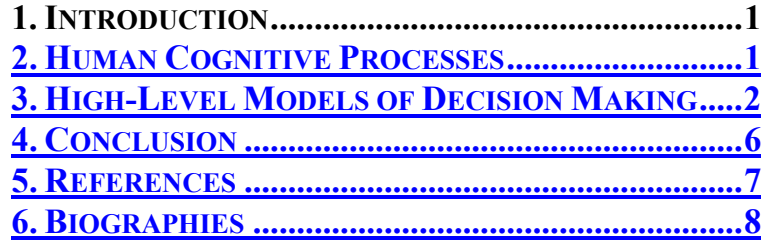

\section{INTRODUCTION}

Decision making is at the core of human cognitive activity. The process of making good decisions is a long studied art that has drawn from previous human experience and been condensed into a number of decision making models. It is not the case that one model dominates all others, or represents an approach that should always be applied to each situation. Many decision making models share common aspects and attributes but differ in the order, area of emphasis, or underlying assumptions. For situations where there is ample time to consider and analyze the situation before making a decision, analytical models can be considered appropriate. However, for military command and control applications during an actual battle, time is limited and uncertainty is high.

This paper reviews a number of decision making models, with primary emphasis on time-critical decision making in a

\footnotetext{
1

${ }^{1} 0-7803-9546-8 / 06 / \$ 20.00 \bigodot 2006$ IEEE

2 IEEEAC paper \#1650, Version 3, December 23, 2005
}

military context. We begin by addressing the underlying cognitive processes that impact decision making including attention, working memory, and reasoning. We then review several of the most widely used and studied models of decision making. The primary contribution of this review paper is to enable those interested in the topic to quickly understand the main issues and gain access to relevant references.

\section{Human Cognitive Processes}

While there are many different models of decision making, there are underlying cognitive processes and issues that are common to most, if not all, decision making models. Here, we describe key cognitive processes and their implications for the design of decision support and human interaction systems.

From a general cognitive perspective, decision making is the process of selecting a choice or course of action from a set of alternatives. Most models describe the human process of decision making as serial staged processes that include steps centered on information gathering, likelihood estimation, deliberation, and decision selection. Related fields of psychology (and beyond) include human reasoning (e.g., inductive and deductive reasoning) and decision theory (e.g., Luce \& Raiffa's rational choice theory [1], Raiffa's decision analysis [2], von Neumann \& Morgenstern's [3] game theory) as well as more general theories of foundations of probability, utility theory, and logic. These theories and processes are useful in trying to understand human decision making because they attempt to describe how humans execute particular stages of decision making.

\section{Attention and Working Memory}

Since human decision making is not a singular process, it stands to reason that underlying cognitive processes are not singular but, instead, reflect a variety of different cognitive processes, combined and utilized in different ways. Nevertheless, the two fundamental processes that underlie human reasoning and decision making are attention and 
memory [4]. Attention is how the brain, often consciously though sometimes automatically, selects information for cognitive processing. Human memory is the capacity to encode, store, and retrieve information. Attention and memory are general processes that each have functionally specialized subdivisions (e.g., memory can be manifested in auditory working memory, visual iconic memory, and longterm memory). It should be pointed out that attention and memory (working memory, in particular) serve as important bottlenecks in human information processing, so understanding how these processes affect the components of reasoning and decision making are vital to developing decision support technologies.

In general, working memory refers to a variety of processes used to maintain mental information in a highly accessible state. Sometimes informally called short-term memory, working memory is closely connected to attention as the limitations in working memory are attributed to specific spatial, temporal, and effort-related characteristics of attention. Working memory should be thought of as a temporary store where conscious, effortful (requiring attention) internal computations are performed. Working memory is also closely related to what is referred to as the 'executive control' [5], the conscious ability to switch between effective task sets, contexts, and intentions.

\section{Reasoning}

Human decision making processes are facilitated using a variety of different reasoning techniques. One such technique is analogical reasoning (inferring novel solutions via analogy to known solutions/methods). Decisions are ultimately made (and thus facilitated) by reasoning about the problem and possible outcomes by making inferences about other decision making events. Many of the theories and techniques for understanding analogical reasoning outlined below are directly relevant for understanding decision making more generally.

Component processes of analogical reasoning include the following serial procedures [6]:

1. Encoding: Translating stimuli to internal (mental) representations

2. Inference: Determining the relationship between problems

3. Mapping: Determining correspondences between new and old items

4. Application: Execution of the decision process

5. Response: indicating the outcome of the reasoning process

Since the steps in this reasoning process proceed in a serial manner, temporal ordering and timing of decision support is critical to improving time-critical decision making. Further analyses shows that reaction times and error rates increase for more complex encodings. Regardless of the stimuli, the encoding step is the largest single component of the reasoning process, taking $\sim 45 \%$ of the overall reasoning time. For example, the encoding of words takes longer than the encoding of schematic pictures, implying that reducing text in displays will facilitate faster decision making. Thus, in general, time critical decision making displays should concentrate on facilitating quicker encoding, possibly by more intuitive symbology and tasking.

Attribute comparison (inference, mapping, application) maps reasonably onto the enumeration and probability estimation performed in the middle steps of the decision making models previously described.

Encoding, inference, and mapping steps vary most by stimuli, suggesting that application specific component taskanalysis should address the most time-consuming components of the decision making process. Componentprocess testing procedures should be utilized both early during spiral development to ensure the decision support systems employ symbols and visual structure to address the most relevant components of the decision making process

\section{High-Level Models of Decision MaKing}

\section{OODA Loop}

One of the best-known decision making models in the military is the OODA loop, created by John Boyd [7]. The OODA loop stands for the following four steps:

1. Observation: take in observations of the overall situation

2. Orientation: make judgments of the situation to understand what it means

3. Decision

4. Action: execute and monitor the decision

A basic strategy for defeating an enemy's command and control (C2) is referred to as "getting inside his OODA loop" by executing your own OODA loops faster than the enemy can. Therefore, the enemy $\mathrm{C} 2$ falls behind in its understanding of the situation and makes decisions based upon old and inaccurate situation assessments, and therefore becomes ineffective. Simply increasing the rate of decision making is not sufficient to achieve this goal. The decisions must also be timely and appropriate to achieve the desired effect.

Recent research has compared the high-stakes, rapid decision making by futures traders to Marines in combat [8]. The traders' equivalent to the OODA loop is ISAA: Information, Sort by Priority, Act, and Assess. One tactic used by the traders with the ISAA loop is to sort trade 
$\begin{array}{llll}\text { Observe } & \text { Orient } & \text { Decide }\end{array}$

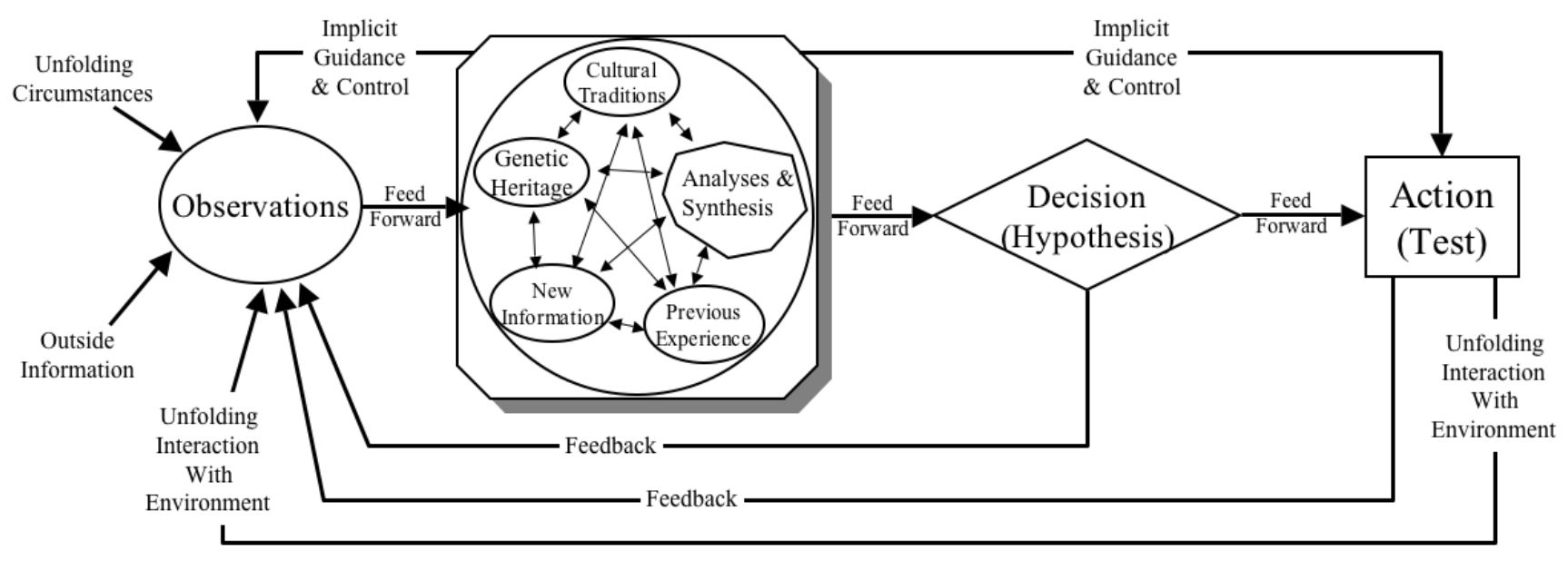

Figure 1 - John Boyd's OODA Loop.

orders by priority based upon experience, then first execute market orders with small positions before moving to larger trades. The results of the initial trades either confirm or refute the trader's understanding of the market, allowing the trader to readjust if the market does not respond as anticipated (a naturalistic strategy as discussed later.)

\section{Kill Chain Model}

More recently, the Air Force has stressed the "kill chain" model, which describes the steps needed to find, recognize, and prosecute mobile targets [9]. The goal is to reduce the time needed to complete the cycle, because if execution is too slow (as it was in Desert Storm) then the kill chain does not work. The mobile targets are able to "shoot and scoot" before the kill chain can be completed. The kill chain steps are summarized in the acronym F2T2EA:
1. Find
2. Fix
3. Track
4. Target
5. Engage
6. Assess

A specific requirement for the "kill chain" is the need to have assets that are physically capable of striking a target on short notice. Without that, even reducing the latency in the decision cycle to zero will not solve the problem. This puts a premium on platforms with long range (e.g., artillery, rockets and air power) and long duration (e.g., unmanned air vehicle platforms that can maintain on station for many hours).

\section{Headquarters Effectiveness Assessment Tool}

In the 1980's, the Headquarters Effectiveness Assessment Tool was developed to reflect $\mathrm{C} 2$ operations [10]. The six steps in this model (see Figure 2) are:

1. Monitor

2. Understand
3. Develop Alternatives

4. Predict

5. Decide

6. Direct

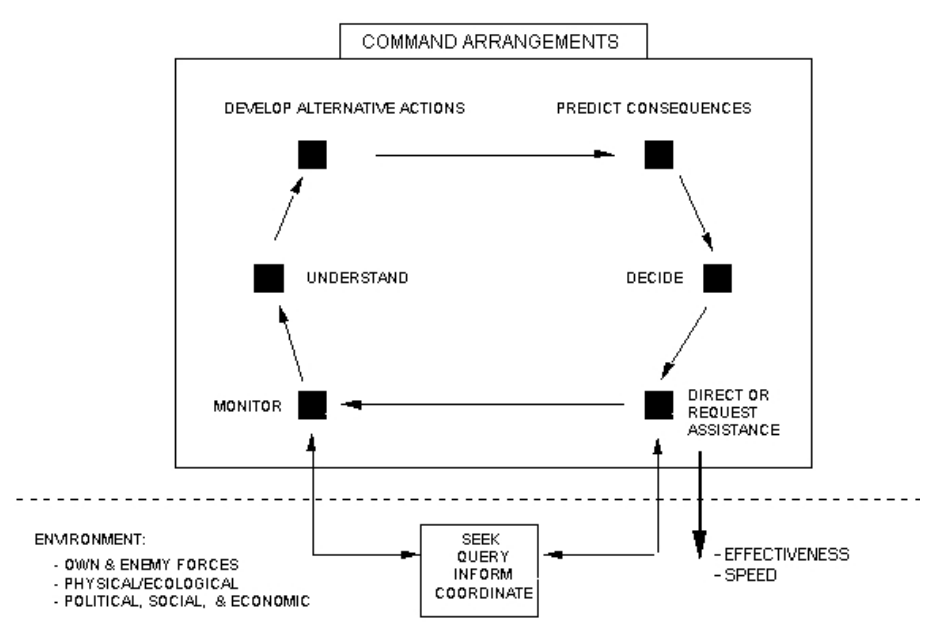

Figure 2. Hayes' Headquarters Effectiveness Assessment

This model explicitly points out that commanders commonly skip the middle two steps (see the discussion of analytical vs. naturalistic models later in this section). The first two steps are considered Battlespace Awareness, which collects facts and produces an understanding of the situation, while the last two steps are Battlespace Management, in which commanders make decisions and disseminate them to forces for execution. Decision Making encompasses the middle four steps.

\section{Triage Models}

Simon in The New Science of Management Decision models the decision making process in three high-level stages [11]:

1. Intelligence: Fact finding, problem and opportunity sensing, exploration. 


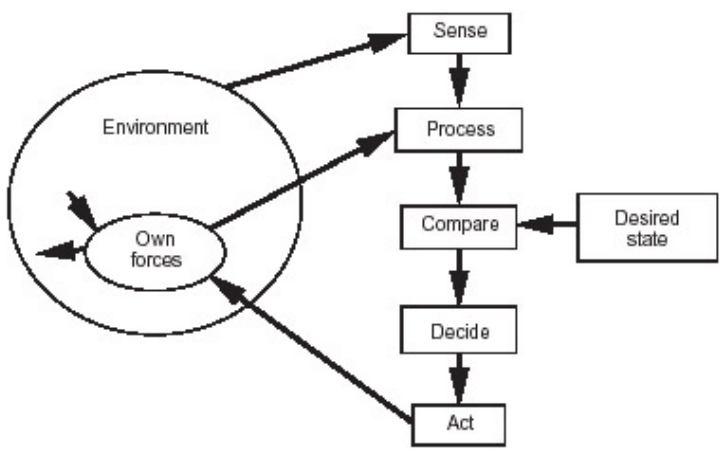

Figure 3. Lawson's control theory model.

2. Design: Formulation of solutions and generation of alternatives.

3. Choice: Decision making, goal maximization, and implementation.

Triage models dissect the problem into key questions to answer, such as in the GO-FITE-WIN acronym. It reminds decision makers of pertinent ways of think of the problem, specifically:

- What are the Goals and Obstacles of thinking?

- How Familiar is the situation?

- How Important is it?

- How much Time is available?

- How much Effort is required for an acceptable level of effort?

- What's Important Now?

Control Theory Based

J.S. Lawson drew a model of $\mathrm{C} 2$ that essentially reflects a control theory mindset [12]. The $\mathrm{C} 2$ process is supposed to sense the state and compare that against some "desired

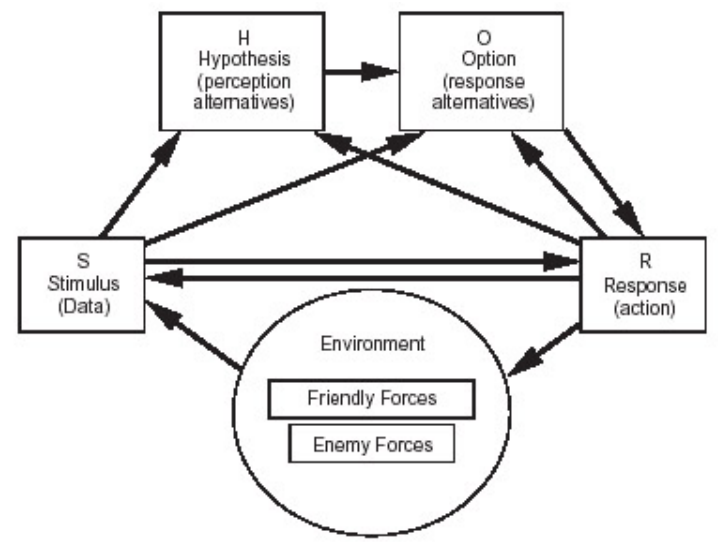

Figure 4. Wohl's SHOR model.

state," then execute actions designed to bring the current state closer to the desired. Figure 3 shows the steps in Lawson's model.

\section{The SHOR Model}

J.G. Wohl sketched a non-linear model of decision making that seems inspired by behavioral or experimental

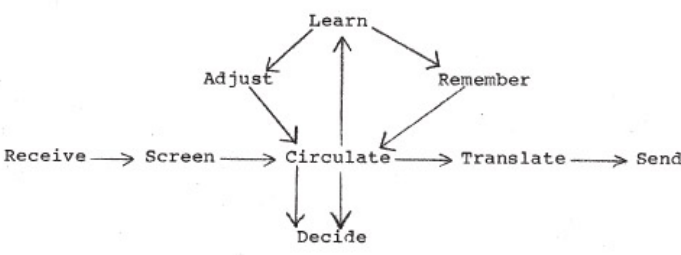

Figure 5. Gorman's description of command functions.

mechanisms. The model is called SHOR, for Stimulus, Hypothesis, Option and Response [13]. Figure 4 shows that the decision maker is not constrained to operate in a particular cycle iterating between steps in a fixed order. Similarly, in a 1980 position paper "A Command Post is not a Place," General Paul Gorman sketched command system functions in a diagram shown in Figure 5 [14].

\section{The Rational Decision Making Model}

Beyond these specific models, there are two philosophical approaches toward decision making: the rational (or logical or analytical) approach vs. the naturalistic (or action-based or recognition-primed) approach.

The Rational decision-making model assumes that a clear set of alternate choices can be generated and their likely outcomes predicted with a significant degree of confidence. It relies heavily on experience or past results to generate the predicted outcomes, and that the information that the decision is based upon is reliable. This model presumes to be objective, by establishing criteria, weighting them, and then choosing the best "score" or highest utility. One example set of steps in this model is:

1. Set organizational goals and objectives

2. Develop alternatives

3. Compare/evaluate alternatives using objective criteria and weights

4. Choose among the alternatives the one that best matches criteria

5. Implement decision

6. Command, lead, manage

7. Feedback loop; observe results and start over again The Rational or Analytic model is a classic approach for decision making and maps well to implementation on a computer since it is highly numeric. It is a linear model that is not dynamic. The claim of objectivity is weak since humans must set the criteria and weights; if these criteria and weights are not reasonable or correct, then the output of this decision process will also be suspect. Variations of this analytical model are called Multi-Attribute Utility Analysis (MAUA, which generates decision matrices)[15] and Decision Analysis [16].

An extreme example of the analytic approach [17] describes a theoretical basis for time-critical applications where the benefits of decisions diminish rapidly as the time it takes to make the correct decision grows. Specifically, this 
approach was applied to monitoring space shuttle propulsion systems, where if a problem is not detected and properly acted upon within several seconds it could result in the loss of a shuttle. They use a decision-theoretic model that processes the large stream of information sent via telemetry, and through probabilistic reasoning, provides the best estimate of what the status or problem is and what the most pertinent data supporting that conclusion are. It is based upon a highly numeric and utility-based metric. However, this model is difficult to use for the military C2 application since for Horvitz's application, both the data and the underlying process and failure mechanisms are well known and understood. There is far more uncertainty in all aspects in military $\mathrm{C} 2$.

Other human-to-human related fields such business and clinical psychology use models that are related to the analytical approach and may provide insights into cognitive processing of decision making [18]. For example, one five step model of psychologically-based decision making is designed from the concepts of classical decision theory [19]. The five basic stages include:

1. Listing of all possible orthogonal or unique actions or alternatives

2. Listing of all possible outcomes for each alternative

3. Estimation of probabilities / likelihoods for each outcome

4. Determination of the desirability based on costs and ramifications of each outcome

5. Application of a decision criterion that yields the best outcome (e.g., selecting the alternative with the highest expected utility).

While relatively simple, this theoretical model provides a straightforward computational approach that approximates commonly used Bayesian or other probabilistic models of action assessment.

Another, slightly more explicit psychology-based model uses eight stages of adaptive decision-making centered on human behaviors or goal states [20], [21]. Components of this model include:

1. Generation of all possible courses of action

2. Gathering relevant information about feasible alternative courses of action

3. Estimation of the probability of success in each alternative, based on experience of other events and projections of current trends

4. Consideration of which goals may be enhanced or diminished for each alternative

5. Deliberation and weighting of facts, probable outcomes, and values for each course of action

6. Elimination of the least favorite course of action

7. Formulation of a tentative plan of action subject to new developments and opportunities
8. Generalization of the decision making process so that it may be employed for future problems.

Rational models can work well for situations that are not time critical and are amenable to numeric analysis. However they often do not map well to how experts actually make decisions under time pressure. The purely analytic model has no dynamic component, preventing evolution over time. Furthermore, it compresses the inherent uncertainty into probabilities or weightings, which may obscure other important sources of uncertainty such as unreliable assumptions, gaps in reasoning, or conflicting evidence.

\section{The Naturalistic Decision Making Model}

The Action-based or Naturalistic model has a different philosophy [22], [23], [24]. It is based upon imposing an interpretation upon an ambiguous situation. The leader takes actions to understand the world, rather than passively collecting information. This approach believes that action and knowledge are inherently linked. There is an inherent assumption that after a point, too much information can be detrimental. This model assumes that knowledge results from actions, from observing consequences. It does not attempt to come up with an ideal or optimal solution. In fact, it assumes that decisions that result in mistakes can be corrected and refined in the future. The Naturalistic model assumes it is not feasible to fully quantify the situation and find a solution mathematically. This approach also differs from others in not relying upon experience, and may be the best if the present situation is very different from any past situations. This model is proactive rather than reactive. Examples of Naturalistic or Action-based decision making include: brainstorming, building and trying many prototypes, and immersion into a foreign environment. Note that this model is the OODA loop in a different order. It becomes the DAOO loop: Decide, Act, Observe, Orient. Rational decision processes are outcome oriented, while naturalistic decision making strategies are process oriented.

Gary Klein describes the Recognition-Primed (or intuitive) decision-making model in his books titled "Sources of Power" and "Intuition at Work" [25], [26]. This model assumes experience is the primary source of wisdom in decision making, and results from many observations and studies of real-life cases of people actually making timecritical decisions in the real world, rather than in laboratory conditions. It generally applies to crisis situations where time is very limited, such as an emergency room or fire fighting, rather than situations where time is not limited and careful consideration is possible. The decision maker reaches and executes the first workable solution rather than attempting to spend a lot of time evaluating many different solutions to find an "optimal" one. This decision might be based upon fragmentary evidence, based upon an expert's 
ability to identify the key symptom, measurement, or aspect that must be determined before making the decision. The decision maker recognizes the situation based upon four features: expectancies, plausible goals, relevant cues, and typical action. The general process of making a decision follows one of these three steps:

1. If the situation matches a typical situation, the decision maker chooses a traditional solution that applies to this typical situation that has worked in the past.

2. If the situation is not typical, the decision maker attempts to get more information in order to map it to one from previous experience.

3. If the situation is novel, the decision maker picks the best action based on experience and implements that. If that doesn't work, he switches to the next best action and so forth.

This process does not compare solutions against each other, but solutions against the situation in a serial fashion. The goal is a "good enough" solution, not the ideal one. The decision-maker is biased toward acting now, not waiting for a complete analysis or knowledge, and doing quick mental

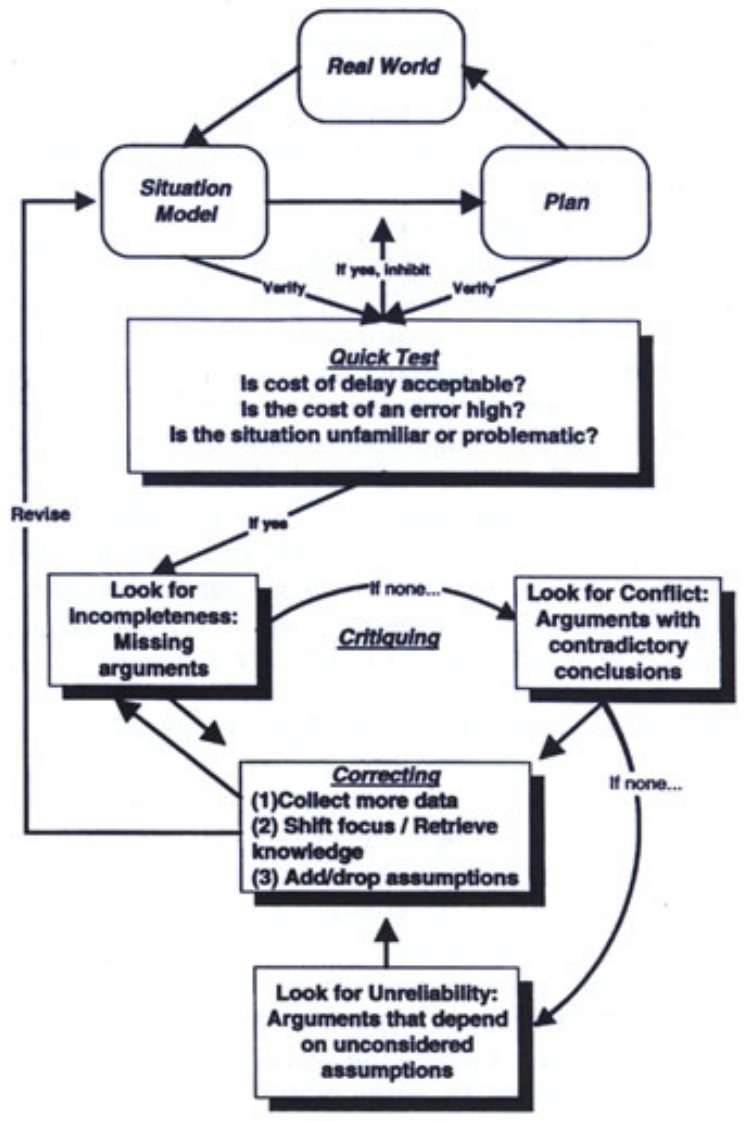

Figure 6. R/M decision model.

evaluations of the feasibility of potential solutions. This model assumes experience is valuable, which means the past is a good predictor of the future. This may not always be true, especially with changing military technologies (classic example: continued use of Napoleonic massed infantry charges against machine gun positions in World War I). This might be more appropriate for smaller, tactical situations than strategic ones where the situation may be too complex for one person to mentally digest and simulate in his head.

Cohen, Freeman and Wolf described a model they call R/M (recognition and metacognition) [27]. The steps in the R/M model are shown in Figure 6. It is a combination of the recognition-primed style of decision making (for routine situations) with a process for improving results of novel situations by probing for flaws and weaknesses and evaluating the results (metacognition). These critiques search for problems of incompleteness, conflict, or unreliability. Incompleteness means the argument does not either support or reject a conclusion of interest. Two arguments conflict if they simultaneously provide evidence both for and against a conclusion of interest. An argument is unreliable if the support it provides depends on unexamined assumptions. Metarecognition has been described as the "observation of one's intuitive practicebased behavior with an eye to challenging and perhaps improving intuition without replacing it."

The R/M (recognition / metacognition) model (see Figure 6) is a dynamic and iterative problem-solving strategy. The next step is determined by the results of the earlier steps, rather than a "global optimization" represented by the rational method. It incrementally generates new hypotheses, tests, and goals. It reconciles pattern recognition with problem solving strategies, combining both use of experience to deal with routine decisions while also having an approach for handling uncertainty and novelty. As such, it appears to be one of the most advanced models of time-critical decision making. It is an example of what Holyoak calls adaptive expertise [28], where an expert has both deep domain knowledge but flexibility on his decision processes and structures to analyze and determine when those do and don't work (i.e., active learning and metacognition are key factors).

\section{Team-Based Decision Making}

Another type of decision making model is team-based decision making. These concepts are less well developed than the decision making models for individuals described above. Some tasks and applications require decision making by a team rather than by a single commander, or that the team members work in tight coordination and interdependence. Examples include a Naval CIC, sports teams, fire fighters, surgical teams, SWAT teams, and cockpit crews. One goal in team-based decision making is implicit coordination: the state where team members can coordinate without overt communication, almost as if they 
could read each other's minds [29]. One factor in establishing implicit coordination is having deep, shared understanding amongst the team members, in the form of shared mental models. Researchers have hypothesized that one strategy for enabling shared mental models is to do cross training, so that all team members are better aware of each other's tasks, responsibilities, and duties [30], [31]. This allows team members to better form expectations and predictions of other members' needs and actions. When team members must coordinate, there is a price because typically the team can only focus attention on one aspect at a time, which they can split their attention amongst team members when they are not actively communicating with each other.

\section{Conclusion}

We have reviewed key cognitive aspects relevant to decision making, and a number of the leading high-level models for time-critical decision making. Clearly, it is not the case that one model dominates all others, or represents an approach that should always be applied to each situation. Also, many of them share common aspects and attributes but differ in the order, area of emphasis, or underlying assumptions. For situations where there is ample time to consider and analyze the situation before making a decision, the analytical model can be considered appropriate. However, for military C2 applications during an actual battle, time is limited and uncertainty is high. Standard U.S. doctrine for such time-critical decision making favors the intuitive (or recognition-primed or naturalistic) approach over the analytical. This belief partially stems from the fact that each situation encountered is unique in some way and there is no perfect solution, so commanders should not agonize to find the ideal solution nor unreasonably dwell upon mistakes or changes. A famous quote from General George Patton perhaps summarizes it best: "A good plan violently executed now is better than a perfect plan next week."

\section{Acknowledgements}

This work was funded in part by Boeing Phantom Works. The authors thank Dr. Anthony Majoros for his support.

\section{REFERENCES}

[1] Luce, R.D. and H. Raiffa, "Games and decisions: introduction and critical survey," New York:Wiley, 1957.

[2] Raiffa, H., "Decision Analysis," Addison-Wesley, 1968.

[3] von Neumann, J. and O. Morgenstern, "Theory of Games and Economic Behavior," Princeton University
Press, 1944.

[4] Newell, A. and H. Simon, "Human Problem Solving." Oxford, England: Prentice-Hall, 1972.

[5] Baddeley, A. D., "Working memory," Oxford, England: Oxford University Press, 1986.

[6] Sternberg, R., Psychological Review, 84(4), pp. 353$378,1977$.

[7] Boyd, J.R., “The Essence of Winning and Losing," see http://www.d-n-i.net/fcs/ppt/boyds_ooda_loop.ppt, 1996.

[8] West, F.J. "War in the Pits: Marine-Futures Traders Wargame" in issue 61 of the National Defense University Strategic Forum, Feb. 1996.

[9] Gobel, Dan, "RF Jamming: The First 100 Years," Journal of Electronic Defense, pp. 45-51, February 1999.

[10] Hayes, R. E., M. Hainline, C. Strack, and D. Bucioni, "Theater Headquarters Effectiveness: It's Measurement and Relationship to Size Structure, Functions, and Linkage," McLean, VA: Defense Systems, Inc, 1983. See also Hayes, Richard E., Conrad Strack, and Daniel Bucioni. (1983b). Headquarters Effectiveness Program Summary. McLean, VA: Defense Systems, Inc.

[11] Simon, H., "The New Science of Management Decision." Upper Saddle River, New Jersey: Prentice Hall PTR, 1977.

[12] Lawson, J.S., "Command and Control as a Process" IEEE Control Systems Magazine, March 1981.

[13] Wohl, J.G., "Force Management Decision Requirements for Air Force Tactical Command and Control," IEEE Transactions on Systems, Man, and Cybernetics, vol. SMC-11, No. 9, Sept. 1981.

[14] Gorman, P., "A Command Post is not a Place," see http://cpof.ida.org/, 1980.

[15] Keeney, R.L. and H. Raiffa, "Decisions with Multiple Objective,” John Wiley \& Sons, Inc., 1976.

[16] Anderson, J., "Cognitive Architectures in a rational analysis," In K. VanLehn (ed.), Architectures for Intelligence, pp. 1-24, Lawrence Erlbaum Associates, Hillsdale, N.J, 1991.

[17] Horvitz, E. and M. Barry, "Display of information for time-critical decision making," In Proceedings of the Eleventh Conference on Uncertainty in Artificial 
Intelligence, Montreal, Canada, Morgan Kaufmann, San Francisco, pages 296-305, August 1995.

[18] John J. Horan (1979). Counseling for effective decision-making: A cognitive-behavioral perspective. MA: Duxbury, North Scituate.

[19] Bross, I.D., "Design for Decision," The Macmillan Company, New York, 1953.

[20] Krumboltz, J. D., \& Baker, R. D., "Behavioral counseling for vocational decisions," In H. Borow (Ed.). Career guidance for a new age. Boston: Houghton Mifflin, 1973.

[21] Krumboltz, J. D., \& Thoresen, C. E., "The effect of behavioral counseling in group and individual settings on information-seeking behavior," Journal of Counseling Psychology, 11, 324-333. 1964.

[22] Pascual, R. and S. Henderson, "Evidence of Naturalistic Decision Making in Command and Control" in C. Zsambok and G. Klein (eds.), Naturalistic Decision Making, Hillsdale, NJ: Lawrence Erlbaum Associates, 1996.

[23] Ogilvie, D.T., "Action-based Naturalistic Models," from "Strategic Decision-making in the Information Age," by Lt. Col. S. A. Shambach, U.S. Army Report on the Strategic Leadership Workshop, Army War College, October 1-2, 1996.

[24] Ogilvie, D., and F. Hauge, "Strategic Decision Making in the 21st Century Army: A Creative Action-Based Approach,” In Stephen J. Havlovic (Ed.) Fifty-eight Annual Academy of Management Best Paper Proceedings 1998, San Diego, CA, August 1998.

[25] Klein, G., "Sources of Power," MIT Press, ISBN 0262-61146-5-2, 1999.

[26] Klein, Gary, "Intuition at Work," Doubleday, ISBN 0385-50288-5, 2003.

[27] Cohen, M.S., J.T. Freeman, and S. Wolf, "Metarecognition in time stressed decision making: Recognizing, critiquing, and correcting," Human Factors vol. 38, pp. 206$219,1996$.

[28] Holyoak K.J., "Symbolic connectionism: Toward third generation

theories of expertise," in K A Ericsson and J Smith (eds), "Toward a general

theory of expertise," New York, Cambridge University Press, pp. 301-312, 1991.
[29] Serfaty, D. and E.E. Entin, "Adaptation to stress in team decision making and coordination," Proceedings of the Human Factors and Ergonomics Society 37th Annual Meeting, Human Factors and Ergonomics Society, Vol. 2, pp. 1228-1232, 1993.

[30] Cannon-Bowers, J. A., E. Salas, and S.A. Converse, S. A., "Shared mental models in expert team decision making," In N. J. Castellan, Jr. (Ed.), "Individual and group decision making: Current issues," Hillsdale, NJ: LEA., pp. 221-246, 1993.

[31] Cannon-Bowers, J. A. and E. Salas, E., "Individual and team decision making under stress: Theoretical underpinnings," In J. A. Cannon-Bowers \& E. Salas (Eds.), "Making decisions under stress: Implications for individual and team training," Washington, DC: APA Press, pp17-38, 1998.

\section{BIOGRAPHIES}

Ronald Azuma is a Senior Research Staff Computer Scientist at HRL Laboratories in Malibu, California.

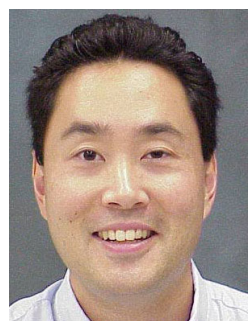

His research interests include Augmented Reality, Virtual Environments, and Visualization. He has a B.S. from the University of California at Berkeley and an M.S. and Ph.D. from the University of North Carolina at Chapel Hill. At HRL, Dr. Azuma has worked on numerous projects in diverse areas, including time-critical decision making, air traffic visualization, and warfighter visualization. He is best known for his contributions to Augmented Reality, including building the first compelling demonstration of that technology and for guiding the field with two survey papers. In 2005, he served as Program coChair for the IEEE ISMAR conference, the premier forum on Mixed and Augmented Reality. He is an IEEE Senior Member.

Mike Daily is a Program Manager in the Information and System Sciences Lab at HRL Laboratories. He has been the principal investigator for a variety of DARPA, DoD, NASA, and internally sponsored programs in intelligent collaboration and visualization, warfighter visualization and interaction, air traffic management, cognitive systems,

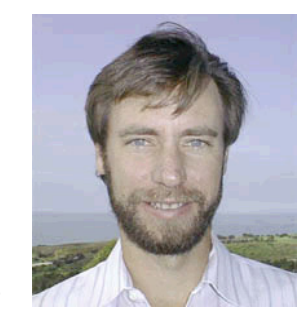
vision-based detection and pose estimation, and automotive crash avoidance. With over 20 years experience, Mr. Daily has researched and published in the fields of distributed virtual environments, 
mobile and wearable interfaces, augmented reality, visualization, collaboration technology, face recognition and human tracking, sensor fusion, robotic perception systems and interfaces, and passive position sensing for human-computer interaction, and holds ten issued patents. He was the principal architect for the HRL perception system that was integral to the first cross-country map and sensor-based navigation of a robotic vehicle in the DARPA ALV program in 1987. He has managed interdisciplinary teams developing the first three-way geographically distributed immersive virtual environment for training in 1997, the first outdoor stabilized augmented reality system in 1999, the first reconfigurable CAVE display in 1999, and the first in-vehicle spatial audio system in 2002. He holds a B.S. in Computer Science from the University of Idaho and an M.S. in Computer Science from the University of New Mexico.

Chris Furmanski is currently developing new ventures in the emerging realm of cognitive and neuro-technology. He previously served as Director of Science and Research for

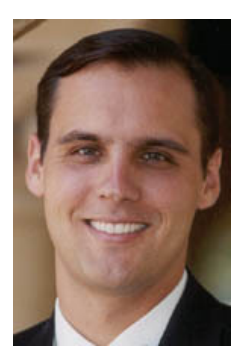

Epoch Innovations in San Francisco, and prior to that was a Research Computer Scientist at HRL Laboratories in Malibu, CA. At HRL, Dr. Furmanski applied principles from cognitive neuroscience to cutting-edge technologies for Boeing, Raytheon, the US Navy, and the Department of Defense (Defense Advanced Research Projects Agency). He adapted theoretical models of human decision making and reasoning into computational models for intelligent software agents, an integral part of several efforts including DARPA's Architectures for Cognitive Information Processing program. 